\title{
The Society for Ecological Restoration
}

\section{I}

ast week I met Mabel, a tireless promoter of conservation and habitat restoration near Houston, Texas. At eighty-nine years of age she moves slowly, but her enthusiasm is undiminished. Cataracts have dimmed her eyesight, but her vision remains clear and determined. Mabel described magical places and remarkable people that forever changed her life. But mostly she described the power of knowledge, enthusiasm, friendships, and collaborations to achieve the goals of conservation and restoration. The collective capabilities and influence of like-minded individuals and organizations can have remarkable and lasting impacts. Although I had planned to share my vision with Mabel, I came away inspired by hers.

In my first editorial as Chair of the Society for Ecological Restoration (SER), I'll briefly describe a few ways SER is spreading knowledge and fostering the friendships and collaborations that promote restoration. The Society for Ecological Restoration seeks "to promote ecological restoration as a means of sustaining the diversity of life on Earth and reestablishing an ecologically healthy relationship between nature and culture." An enthusiastic and knowledgeable membership is our greatest strength, and most of our successes involved collaborations with like-minded organizations. We continue to seek new collaborations - both formal and informal—in pursuit of our collective vision.

\section{Membership}

The Society for Ecological Restoration is blessed with enthusiastic members in over 60 countries_each having unique skill sets, knowledge, and goals. There are many other individuals with knowledge and interest in restoration, but they are not members. We must do a better job of incorporating and benefitting from that enthusiasm and knowledge. As a member of SER, you will learn, foster friendships, and benefit from new collaborations. I encourage you to take advantage of our information and participate in the meetings of our chapters and international meetings. If you are a member-become a more active member. If you are not yet a member-consider becoming a member and providing the benefit of your experience and enthusiasm. View our website at www.ser.org to find our chapters, free publications, news of upcoming events,

Ecological Restoration Vol. 29, No. 3, 2011

ISSN 1522-4740 E-ISSN 1543-4079

(C2011 by the Board of Regents of the University of Wisconsin System. and membership information. We are in the process of a major rebranding effort that will include a new look for our website, but the contents remain focused on restoration.

\section{Providing Restoration Information}

We are proud of our multiple outlets for presenting restoration information and facilitating dialogue among practitioners, scientists, policy-makers, and the interested lay person. SER's relationship with the University of Wisconsin Press and Ecological Restoration is one excellent example of the mutual benefits of strategic collaborations. Our common interest in providing relevant knowledge to restoration practitioners continues to shape our partnership and benefits the restoration community. The journal Restoration Ecology (www.wiley.com/bw/journal.asp? ref=1061-2971), published by Wiley-Blackwell, plays a complementary role by presenting research on restoration and ecological principles that explain restoration processes, describe techniques, and synthesize information on restoration-related topics. In a relatively brief period, Restoration Ecology has gained international recognition for its scientific merit and practical applications. Richard Hobbs, as Editor-In-Chief, and Susan Yates, as Managing Editor, along with their editorial board have done an exceptional job. The Society for Ecological Restoration and Island Press Book series on the Science and Practice of Restoration (www.islandpress. $\mathrm{org} / \mathrm{ser} / \mathrm{index} . \mathrm{html}$ ) has become a rich and varied source of restoration-related information. James Aronson as Series Editor and Karen Holl as Associate Editor have assembled a remarkable restoration-focused book series in collaboration with Barbara Dean at Island Press.

Several online resources are available for download through the SER website. The SER Primer is a concise statement of restoration principles and includes a clear definition of what restoration is, how it is planned, conducted, and evaluated, and how it coordinates with related disciplines. Three long-time SER members (Andre Clewell, John Rieger and John Munro) compiled a valuable resource-Guidelines for Developing and Managing Ecological Restoration Projects. A global rationale for ecological restoration (Ecological Restoration - a means of conserving biodiversity and sustaining livelihoods) is also available.

\section{Conferences}

Attending meetings of SER chapters or the international society has always been a rewarding experience for me. The opportunity to visit with restoration practitioners, 
regulators, consultants, scientists, and volunteers never fails to enlighten and entertain. The opportunities for making like-minded friends and building productive collaborations are immense. I encourage you to visit the SER website to identify conferences, tours, and workshops of benefit to you. As I write this, we are planning a Fourth World Conference on Ecological Restoration to be held in Merida, Mexico, August 21-25, 2011. Unfortunately, that will be over by the time you read this. In 2013, we will celebrate SER's 25 th anniversary by holding our international meeting in Madison, Wisconsin. The dates and details of that meeting are still being determined, but I encourage you to begin making plans to attend.

\section{SER Practitioners Certification Program}

The Society for Ecological Restoration is creating a certification program for practitioners of ecological restoration. Several dedicated members, lead by John Stanley and Keith Bowers, are leading this effort. SER will create a subsidiary organization-SER Practitioners Institute (SERPI) - to administer the certification program. The goals of the certification program are to: 1 ) provide practitioners of ecological restoration with professional credentials; 2 ) improve the quality of ecological restoration projects; 3) foster the incorporation of the principles of ecological restoration, as embodied in the SER foundation documents, into the decision-making process; 4) develop an interactive community of practitioners that are actively engaged in professional improvement; 5) create standards for practitioners of ecological restoration; and 6) stimulate growth of the profession of ecological restoration. Three certification levels will be offered: Certified Ecological Restoration Practitioner In-Training (CERPIT), Certified Ecological Restoration Practitioner (CERP), and Certified Senior Ecological Restoration Practitioner (CSERP). Specific requirements for certification vary depending on the level of certification. SERPI plans on accepting applications for certification in 2012.

Finally, I want to express my sincere appreciation to Jim Harris - former Chair of SER, our hard working SER Board of Directors, and former SER leaders who continue to provide me with strategic and practical guidance. I actively encourage our members to improve our effectiveness through participation at chapter and international levels. I am especially dependent on and grateful to our SER staff for their dedication and continuing efforts.

Steve Whisenant

Chair

Society for Ecological Restoration 